\title{
Construcción y modelado de un prototipo fan $\&$ plate para prácticas de control automático
}

\author{
Cristina Lampón, Javier Martín, Ramon Costa-Castelló \\ Departament d'Enginyeria de Sistemes, Automàtica i Informàtica Industrial (ESAII) \\ Escola Tècnica Superior d'Enginyeria Industrial de Barcelona (ETSEIB) \\ Universitat Politècnica de Catalunya (UPC) \\ cristina.lampon,javier.martin,ramon.costa@upc.edu \\ Muppaneni Lokesh Chowdary \\ School of Mechanical Engineering. SASTRA UNIVERSITY
}

\section{Resumen}

En este trabajo se presenta una plataforma de prácticas de control automático robusto y de bajo coste. El trabajo describe los componentes y la arquitectura propuestos, además de presentar los modelos utilizados para describir su comportamiento. Finalmente se muestran algunos resultados experimentales.

Palabras clave: control automático, práctica de control, modelado, fan and plate.

\section{INTRODUCCIÓN}

Es bien conocido que los trabajos experimentales son uno de los pilares de la educación en el ámbito de la ingeniería y en particular de la ingeniería de control [1]. La realización de prácticas masivas en grupos grandes requiere disponer de numerosos equipos y que estos sean robustos al paso del tiempo y de los estudiantes. Por ello, habitualmente en la mayoría de centros y en particular en la ETSEIB se suelen utilizar equipos comerciales. Estos equipos presentan un coste elevado que en los últimos tiempos, de carestía económica, han sido difíciles de asumir. Por dicho motivo en el departamento de ESAII, y en particular en la sección de la ETSEIB, se han desarrollado diferentes proyectos para construir y adaptar plantas para la realización de prácticas de regulación automática $[2,3]$. Uno de los trabajos realizados en los últimos tiempos ha sido el diseño, desarrollo y modelado de una planta tipo fan and plate $[4,5,6]$. Este tipo de planta es una de las plantas tradicionalmente utilizadas en cursos introductorios de control automático.

El sistema de ventilador y placa presenta, habitualmente, una entrada, que se utiliza para fijar la velocidad de giro del ventilador y una señal de medida que permite obtener el ángulo de la placa. El objetivo de control es fijar el ángulo de la placa a partir de la velocidad de giro del ventilador. Desde un punto de vista de ingeniería de control, esta planta presenta una característica no lineal pues el flujo de aire impacta sobre la placa de ma-

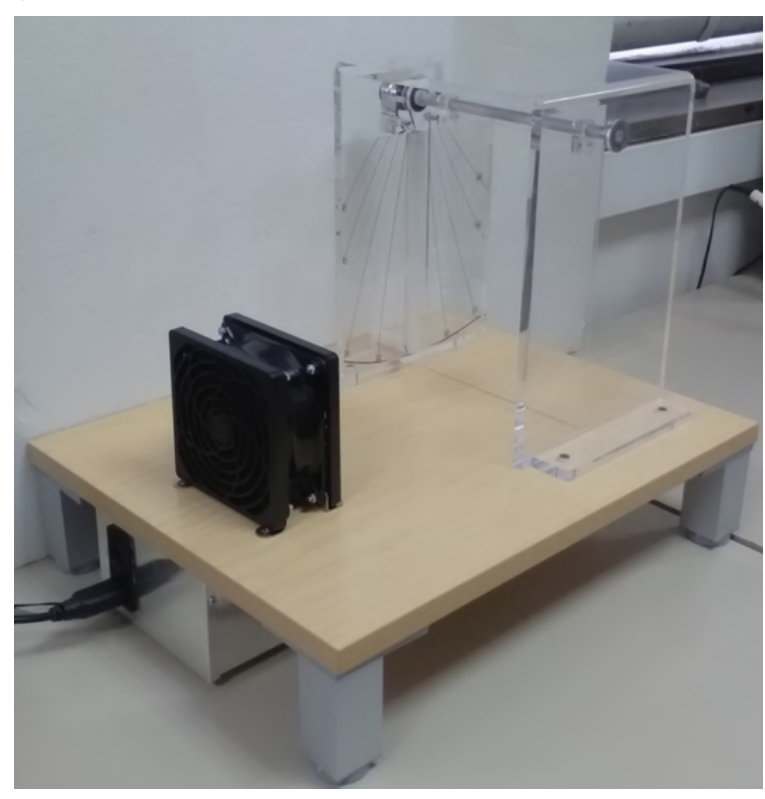

Figura 1: Fotografía del prototipo

nera diferente en función del ángulo. Otra de las características tradicionales de este tipo de planta es que el flujo de aire genera turbulencias que producen oscilaciones sobre la placa, estas turbulencias no son compensables y por tanto inducen limitaciones en el ancho de banda que se puede conseguir.

En este trabajo se aborda el proceso de construcción de un sistema fan and plate robusto y de bajo coste (sección 2), posteriormente se presenta el modelo no lineal y su procedimiento de ajuste paramétrico (sección 3), finalmente se obtiene un modelo linealizado, se diseña un controlador tipo $\mathrm{PI}[7,8]$ y se valida experimentalmente su comportamiento (sección 4). Aunque el modelado se ha realizado principalmente con MATLAB/Simulink se ha implementado también un laboratorio virtual con EJSs [9], de cara a integrar la planta en un laboratorio virtual y remoto.

\section{Descripción del prototipo}

El prototipo diseñado (Figura 1) se compone de una estructura que hace las veces de soporte, un 


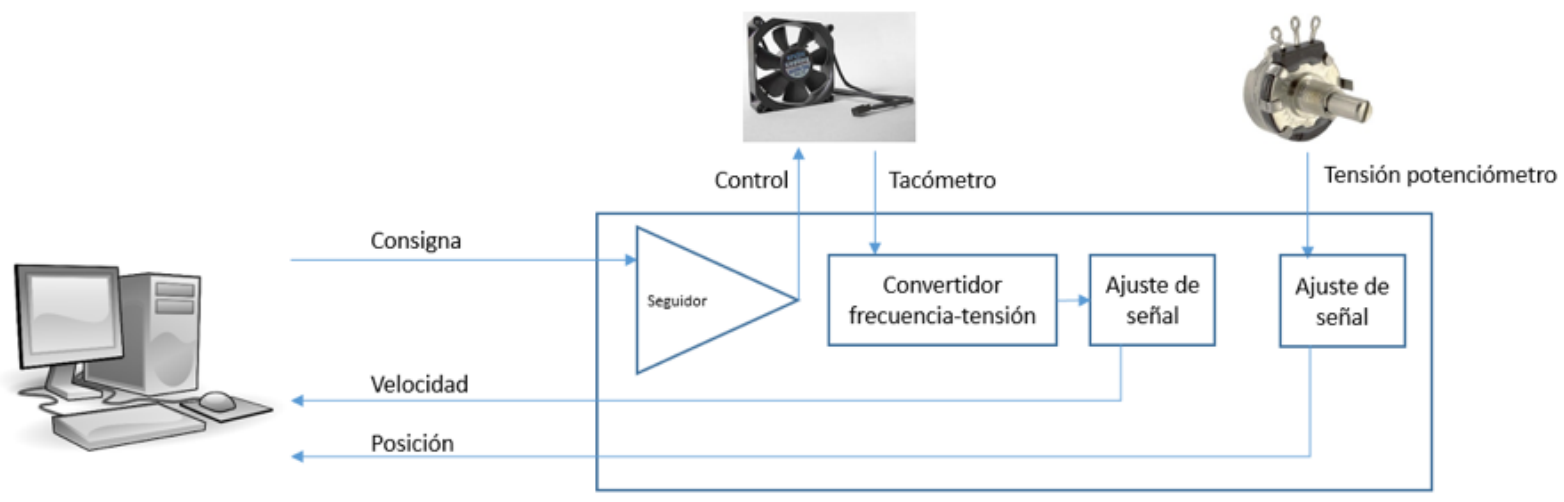

Figura 2: Esquema del sistema utilizado para la realización de las prácticas de control automático

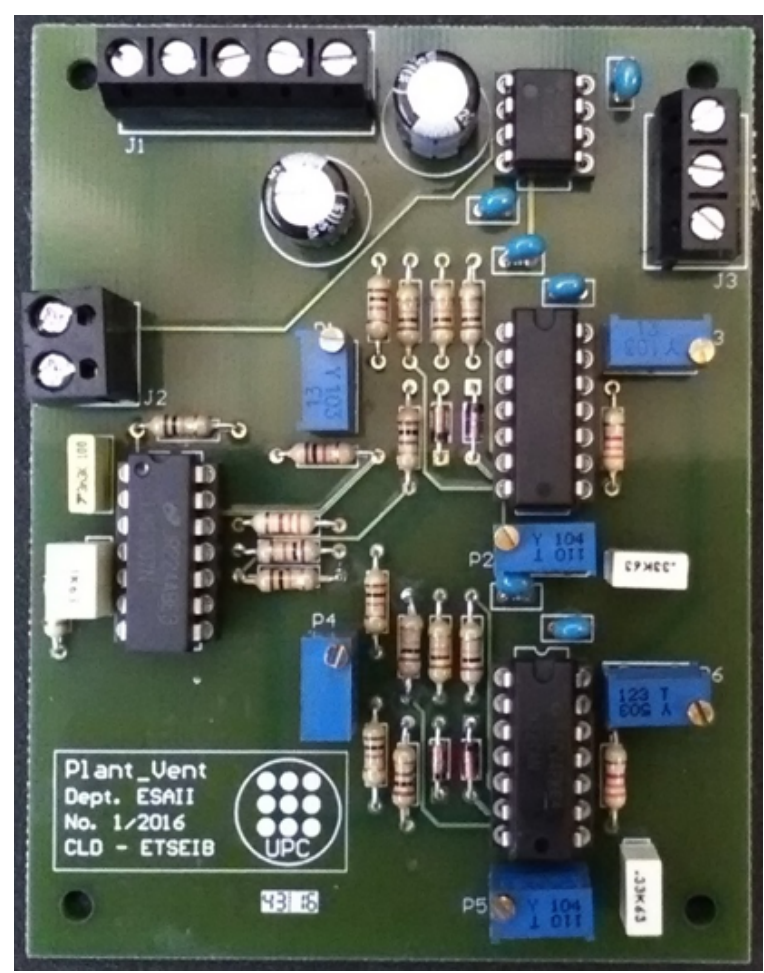

Figura 3: Imagen de la tarjeta electrónica desarrollada para el proyecto.

ventilador, una plancha de metacrilato, un potenciómetro y una caja, conectada a la red eléctrica, que alberga la alimentación del sistema y la electrónica que trata las señales de entrada y salida.

La planta se conecta a la tarjeta de adquisición de datos instalada en el PC mediante un conector SCSI del lado del PC y una regleta del lado de la caja de electrónica de la planta (Figura 2).

El ventilador está fijado a la tabla de la estructura de manera que queda encarado a la plancha y centrado con esta, a $26 \mathrm{~cm}$ de distancia. La plancha se conecta con el eje de giro mediante unos enganches, también de metacrilato. Solidariamente al eje de giro se monta el potenciómetro que proporciona la posición de la plancha. Se ha seleccionado un potenciómetro de baja fricción para que el proceso de medida influya lo mínimo posible en la dinámica del sistema.

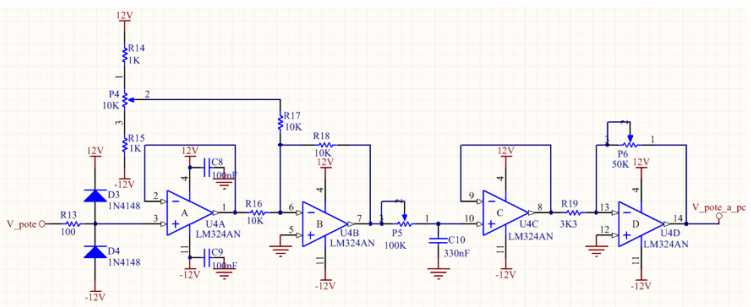

Figura 4: Adaptador de la señal del potenciómetro.

Las patas 1 y 3 del potenciómetro se conectan a $12 \mathrm{~V}$ y $-12 \mathrm{~V}$ respectivamente, tensión proporcionada por la fuente de alimentación contenida en la caja de electrónica de la planta, bajo la tabla de madera. La pata 2 del potenciómetro, el cursor, se conecta a una placa electrónica (Figura 3) -diseñada específicamente para esta aplicaciónpara hacer pasar la señal por un ajuste de rango (Figura 1), cuya salida será la entrada en el PC (mediante la tarjeta ADDA) de la señal de posición del sistema.

Por robustez y simplicidad se ha seleccionado un ventilador industrial que incorpora la electrónica de potencia y una medida de velocidad angular. El ventilador consta de cuatro cables: tensión (rojo), masa (azul), PWM (violeta) y tacho (blanco). Si bien el cable de control recibe la etiqueta PWM por parte del fabricante, este modelo de ventilador permite el control tanto mediante modulación de ancho de pulso como mediante señal analógica de 0 a $5 \mathrm{~V}$, sin necesidad de configuraciones externas específicas. Por simplicidad, para esta planta se utiliza la señal analógica de control de 0 a $5 \mathrm{~V}$. 
El ventilador necesita una alimentación de $24 \mathrm{~V}$, proporcionados por la fuente de alimentación contenida en la caja de electrónica de la planta. La consigna enviada desde el PC entra en la caja de electrónica para pasar por un circuito de seguidor (Figura 6). La salida de este circuito se conecta con la entrada PWM del ventilador.

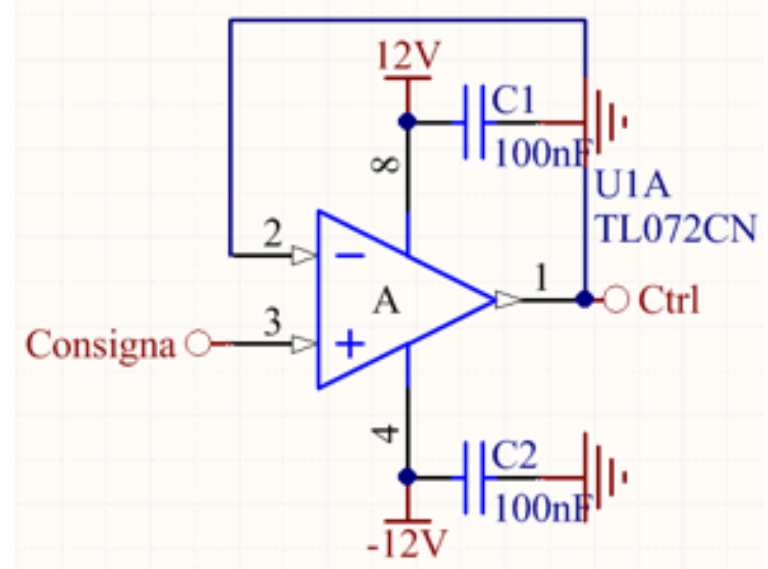

Figura 5: Entrada de consigna y salida de control del ventilador (seguidor de tensión).

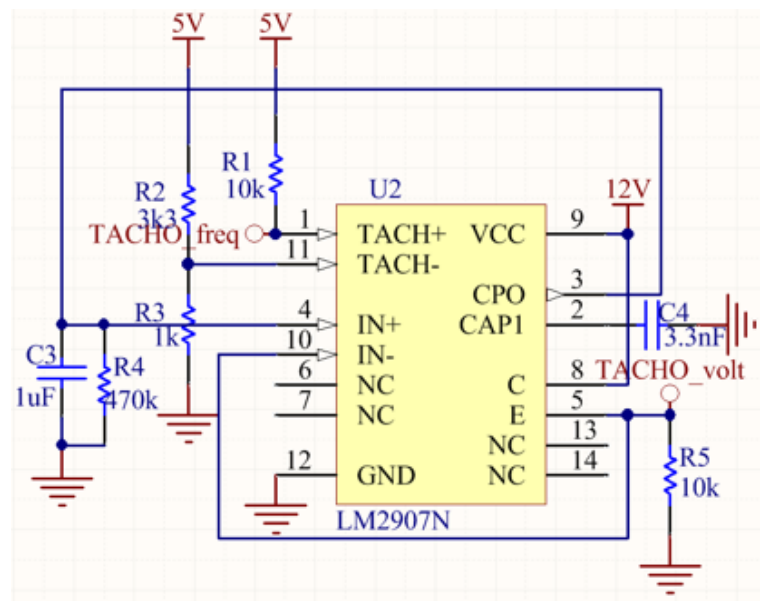

Figura 6: Convertidor frecuencia-tensión utilizado para obtener una medida analógica de la salida de velocidad del ventilador.

Aunque en este trabajo se utiliza un PC corriendo MATLAB/Simulink para realizar las entradas/salidas el sistema está pensado para utilizar hardware de bajo coste para cerrar el lazo de control $[10,11,12,13]$.

La tabla 1 muestra los componentes utilizados en la construcción del prototipo, su marca y su coste económico. Dado que todos los componentes son fáciles de encontrar y presentan características industriales es de esperar que el prototipo sea robusto y soporte bien el paso del tiempo y los estudiantes. Como se puede ver, el coste del prototi-

\begin{tabular}{|l|c|c|c|}
\hline & Modelo & Marca & Importe \\
\hline Fuente de alim. & LPQ202-M & Emerson & $127.53 €$ \\
\hline Potenci. & P-2201-A502 & Novotechnik & $200.74 €$ \\
\hline Ventilador & 4114 N/2H7P & ebmpapst & $114.21 €$ \\
\hline Placa elec. & & & $46.69 €$ \\
\hline Caja & $1444-1593$ & Hammond & $10.15 €$ \\
\hline Estructura & & & $411.52 €$ \\
\hline Total & & & $910.84 €$ \\
\hline
\end{tabular}

Cuadro 1: Componentes y coste de los diferentes componentes del prototipo.

po supera ligeramente los $900 €$ cosa que lo hace muy competitivo en comparación con el precio de las plantas didácticas de características similares. Por ello, se adapta a las características planteadas inicialmente.

\section{Modelado y ajuste}

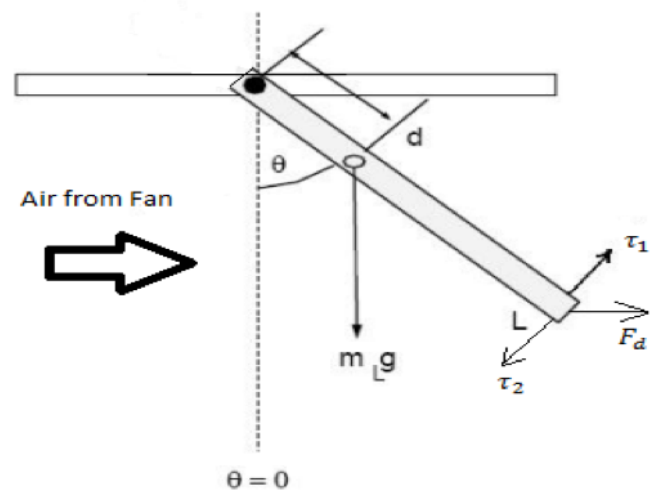

Figura 7: Fuerza aplicada por el flujo de aire sobre la placa.

La fuerza aplicada sobre la placa debido al aire se puede expresar como (Figura 7):

$$
F_{d}=C_{d} \rho A \frac{v^{2}}{2} \cos (\theta)
$$

donde $C_{d}$ es el Coeficiente de fuerza de arrastre (1.28 para el aire), $\rho$ es la densidad del medio $\left(1,225 \frac{\mathrm{kg}}{\mathrm{m}^{3}}\right.$ para el aire), $A$ es la área de la placa $\left(0,3 \cdot 0,2 \mathrm{~m}^{2}\right.$ en el prototipo), $\theta$ es el ángulo de la placa respecto la vertical y $v$ es la velocidad del aire en $\mathrm{m} / \mathrm{s}$. El par producido por esta fuerza sobre el eje de giro corresponde a:

$$
\tau_{1}=F_{d} \cdot L \cdot \sin \left(\frac{\pi}{2}-\theta\right) .
$$

El par producido por la gravedad en el cuerpo se opone al par de fuerza de arrastre, $\tau_{1}, \mathrm{y}$, puesto que nuestro cuerpo se asume uniforme, el centro de la gravedad está exactamente en el centro por 


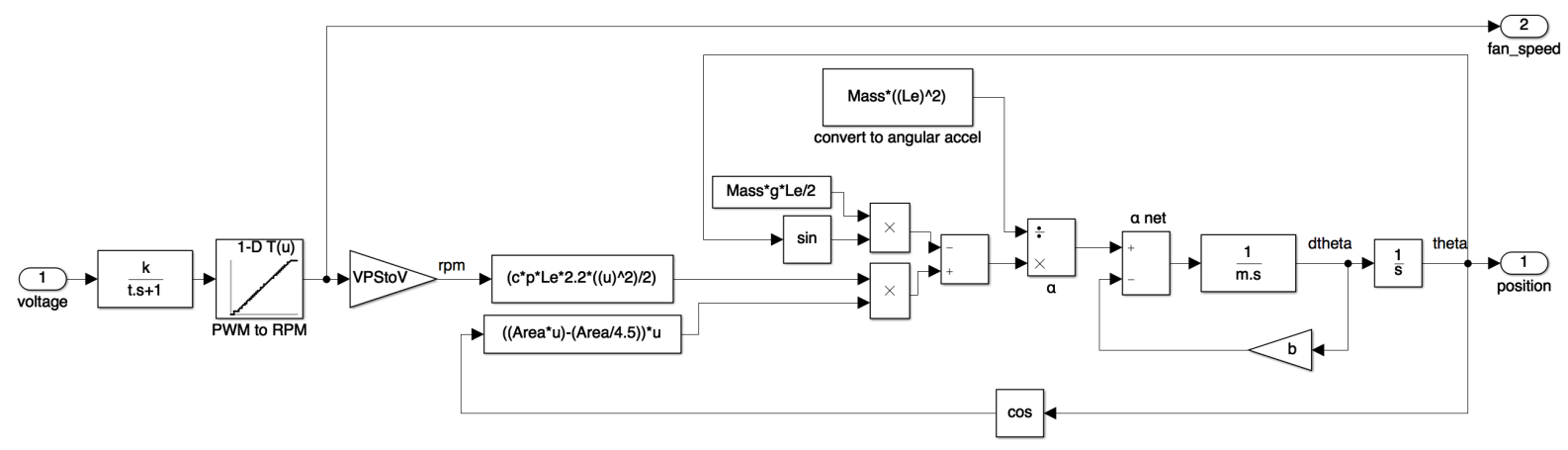

Figura 8: Codificación en MATLAB/Simulink del modelo desarrollado.

lo que:

$$
\tau_{2}=m \cdot g \cdot \frac{1}{2} \sin \theta
$$

donde $m=0,1614 \mathrm{~kg}$ es la masa de la placa y $g=9,81 \frac{\mathrm{m}}{\mathrm{s}^{2}}$ es la constante de gravitación.

El par que actúa sobre el eje de rotación, $\alpha$ corresponde a:

$$
m \cdot r^{2} \cdot=\tau_{1}+\tau_{2} .
$$

Posteriormente este par se utiliza como entrada a un sistema de primer orden que genera la velocidad angular que posteriormente es integrada para generar la posición angular. La Figura 8 muestra la codificación del modelo completo en MATLAB/Simulink.

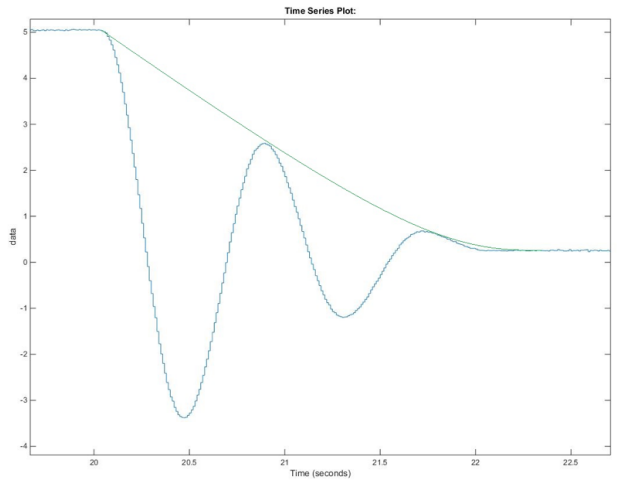

Figura 9: Experimento 1: Oscilación libre. Datos experimentales y envolvente ajustada.

Aunque muchos de los parámetros del modelo pueden obtenerse a partir de la descripción geométrica del sistema o de las propiedades físicas del aire y demás elementos, otros como la fricción son difíciles de conocer a priori. Por ello se han planteado algunos ensayos para calibrar el sistema. En particular, se ubica la placa en un ángulo determinado y se deja oscilar libremente. La Figura 9 muestra un primer experimento. Mediante este ensayo y utilizando la envolvente de la oscilación se puede

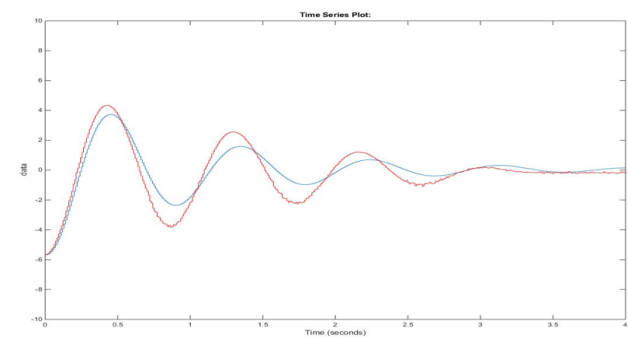

Figura 10: Experimento 2: Oscilación libre. Datos experimentales y salida del modelo.

ajustar un coeficiente de fricción $b=0,5203 \mathrm{~kg} / \mathrm{s}$. La Figura 10 muestra un segundo ensayo y el ajuste que presenta el modelo con los parámetros fijados. Como se puede ver, este valor de $b$ ajusta bastante bien el comportamiento para ángulos iniciales grandes. En la práctica, y dado que el sistema es no lineal y la fricción es un proceso complejo, el coeficiente de fricción ajustado depende de las condiciones iniciales del experimento (amplitud de las oscilaciones). En el resto del trabajo se ha tomado un valor medio que es $b=0,321 \mathrm{~kg} / \mathrm{s}$.

Utilizando el modelo de MATLAB/Simulink (Figura 8) se puede ajustar una curva de comportamiento estacionario, esta se muestra en la figura 12.

La Figura 13 muestra la respuesta temporal de lazo abierto correspondiente al modelo obtenido y a la planta real. Como se puede observar la salida de ambos es muy parecida por lo que se puede concluir que el modelo ajusta la realidad. Puede observarse también que la posición angular del sistema no es exactamente constante, esta presenta oscilaciones alrededor del punto. Estas oscilaciones provienen de las turbulencias creadas por el aire al chocar contra la placa y son intrínsecas al sistema y no pueden ser compensadas. A efectos del controlador pueden modelarse como un ruido aditivo a la salida. 


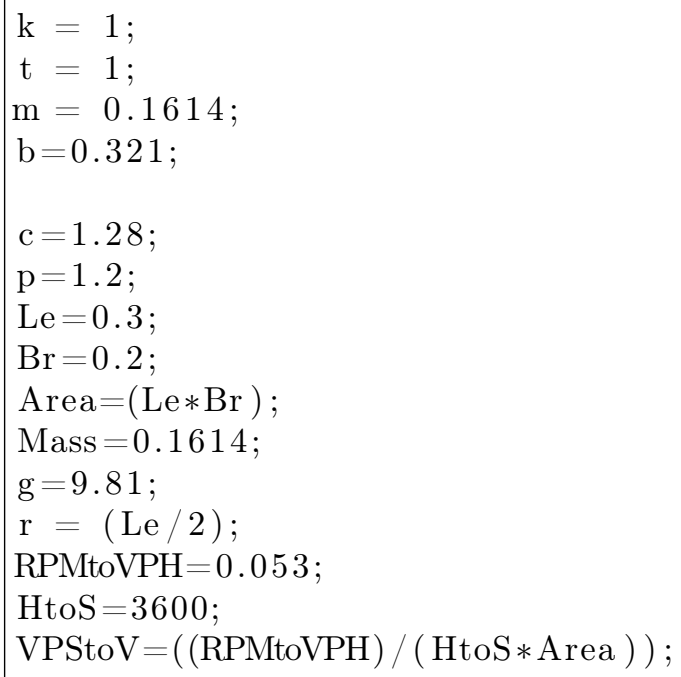

Figura 11: Valor de los parámetros utilizados en el modelo.

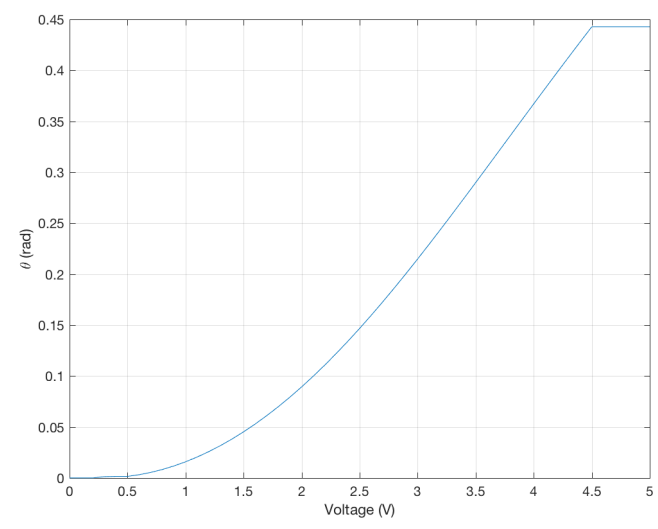

Figura 12: Curva de comportamiento estacionario obtenida del modelo MATLAB/Simulink (Figura 8).

Además del modelo MATLAB/Simulink se ha desarrollado un modelo utilizando EJSs, la Figura 14 muestra la vista de este modelo. Este tipo de modelo permitirá más adelante realizar prácticas virtuales y remotas con este dispositivo.

\section{Diseño de sistema de control}

Aunque el sistema planteado es claramente no lineal, es posible obtener un modelo linealizado alrededor de un punto de equilibrio. Para ello se elige un punto nominal situado en una inclinación de $10^{\circ}$, para este punto es necesario aplicar un voltaje de $2,7117 \mathrm{~V}$. Utilizando MATLAB/Simulink o aplicando una expansión en serie de Taylor de la ecuaciones no lineales [7] es posible obtener el

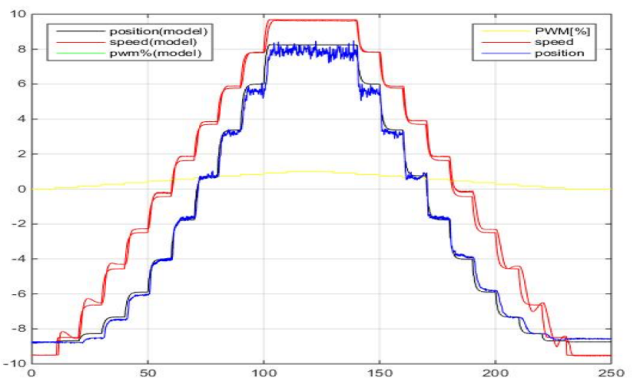

Figura 13: Comportamiento de lazo abierto del sistema. Comparación de la respuesta del modelo (Figura 8) y sistema real.

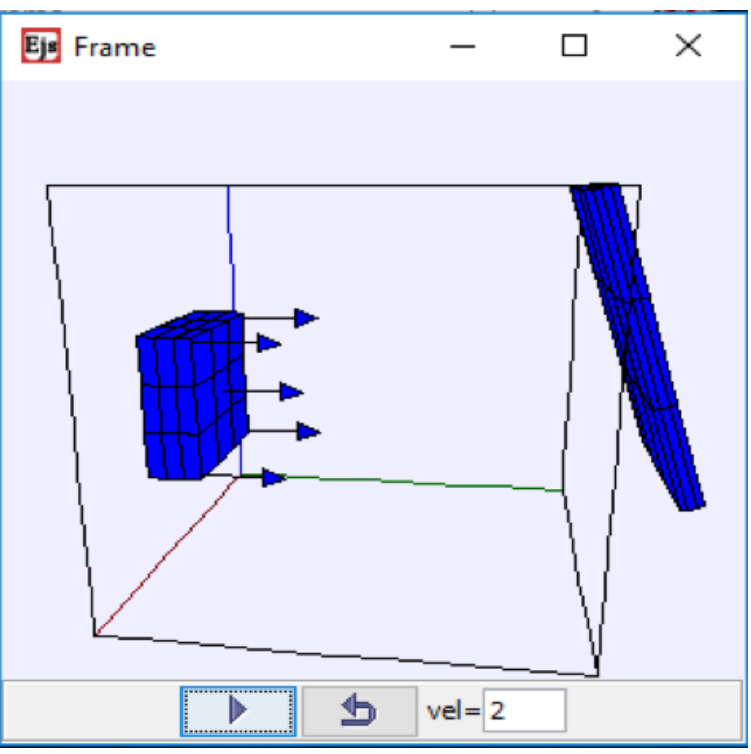

Figura 14: Vista del modelo del sistema desarrollada mediante EJSs.

siguiente modelo:

$$
G(s)=\frac{\Delta \theta(s)}{\Delta u(s)}=\frac{14,45}{s^{3}+2,989 s^{2}+108,9 s+106,9} .
$$

A partir de este modelo se puede diseñar un controlador PI que regule la posición de salida. La figura 15 muestra el comportamiento de lazo cerrado para el modelo y el sistema experimental. Como se puede observar el comportamiento de ambos es bastante parecido cosa que valida el procedimiento desarrollado. Como se puede comprobar, pese a que el controlador se ha diseñado para un punto de equilibrio concreto el sistema de lazo cerrado funciona correctamente en un rango amplio de ángulos.

En asignaturas avanzadas la variación del modelo linealizado puede ser modelado mediante un modelo de incertidumbre no estructurada. 


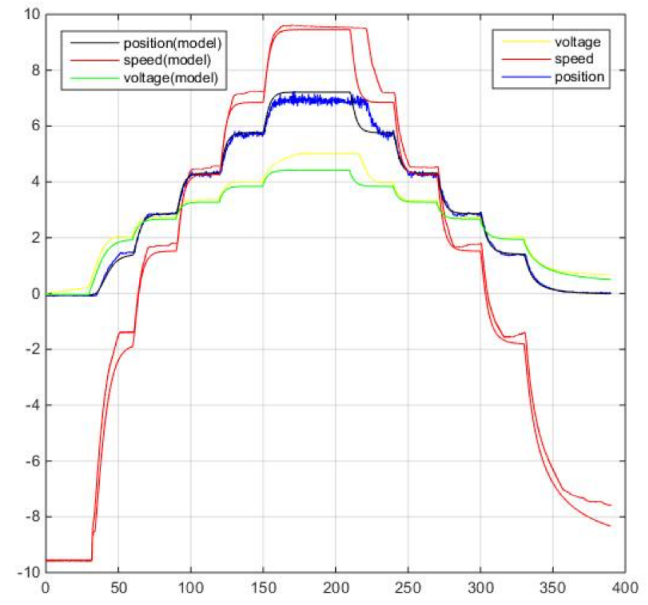

Figura 15: Comportamiento del sistema de lazo cerrado. Utilizando el modelo MATLAB/Simulink (Figura 8) y el sistema real.

\section{Conclusiones}

Se ha presentado un prototipo de planta didáctica tipo "fan and plate" de bajo coste y robusto. En la actualidad el departamento de ESAII está construyendo varios elementos de este tipo con el objetivo de equipar el laboratorio docente.

Se ha ilustrado el procedimiento que habitualmente se sigue en unas prácticas introductorias de control automático. Estas plantas pueden utilizarse en asignaturas avanzadas que plantean técnicas como el control robusto o el control adaptativo.

\section{Agradecimientos}

Este trabajo ha sido parcialmente financiado por el proyecto DPI2015-69286-C3-2-R del Ministerio de Educación de España (MINECO/FEDER).

\section{Referencias}

[1] S.Dormido Bencomo. Control learning: present and future. Annual Reviews in Control, 28(1):115 - 136, 2004.

[2] Cristina Lampón, Ramon Costa-Castelló, and Sebastián Dormido. Hands on laboratory for classical nonlinear control systems : the dead-zone case. In IEEE, editor, 2016 IEEE Conference on Control Applications (CCA), pages 816-820, Buenos Aires, Argentina, Sept 2016. IEEE. ISBN: 978-1-5090-0754-7.

[3] Cristina Lampón, R. Costa-Castelló, and Sebastián Dormido. Módulo didáctico para la realización de experiencias de control no lineal. In Actas de las XXXVII Jornadas de Automática, pages 93-99, Madrid, septiembre 2016. CEA. ISBN: 978-84-617-4298-1.
[4] Arjin Numsomran and Vittaya Tipsuwanporn. The design of robust pid control for fan and plate process. In Proceedings of the Ninth IASTED International Conference on Control and Applications, CA '07, pages 273277, Anaheim, CA, USA, 2007. ACTA Press.

[5] E. Dincel, Y. Yalçin, and S. Kurtulan. A new approach on angular position control of fan and plate system. In 2014 International Conference on Control, Decision and Information Technologies (CoDIT), pages 545-550, Nov 2014.

[6] María Macarena Sevilla, Iñaki Díaz David Bárcena, and Jorge Juan Gil. Control de un sistema no lineal con retraso ventiladorplaca. In Actas de las XXXVI Jornadas de Automática, pages 1042-1049, Bilbao, septiembre 2015. CEA. ISBN 978-84-15914-12-9.

[7] José Luis Guzmán Sánchez, Ramon Costa Castelló, Manuel Berenguel Soria, and Sebastián Dormido Bencomo. Control automático con herramientas interactivas. Pearson, 2012. ISBN: 9788483227503.

[8] Ramon Costa Castelló and Enric Fossas. Sistemes de Control en Temps Discret. Edicions UPC, 2014. ISBN: 978-84-9880-492-8.

[9] Félix J. García Clemente, Francisco Esquembre, and Loo Kang Lawrence Wee. Deployment of physics simulation apps using easy javascript simulations. In 2017 IEEE Global Engineering Education Conference (EDUCON), pages 1093-1096, April 2017.

[10] Adolf Izquierdo Borràs and R. CostaCastelló. Prácticas experimentales de control digital con elementos de bajo coste. In Actas de las XXXVII Jornadas de Automática, pages 402-409, Madrid, septiembre 2016. CEA. ISBN: 978-84-617-4298-1.

[11] R. Barber, M. Horra, and J. Crespo. Practices using simulink with arduino as low cost hardware. IFAC Proceedings Volumes, 46(17):250 - 255, 2013.

[12] F.A. Candelas, G.J. García, S. Puente, J. Pomares, C.A. Jara, J. Pérez, D. Mira, and F. Torres. Experiences on using arduino for laboratory experiments of automatic control and robotics. IFAC-PapersOnLine, 48(29):105 - 110, 2015.

[13] P. Reguera, D. García, M. Domínguez, M.A. Prada, and S. Alonso. A low-cost open source hardware in control education. case study: Arduino-feedback ms-150. IFACPapersOnLine, 48(29):117 - 122, 2015. 